\title{
Lipoprotein Particles Cross the Blood-Brain Barrier in Drosophila
}

\author{
Marko Brankatschk and Suzanne Eaton \\ Max Planck Institute of Molecular Cell Biology and Genetics, 01307 Dresden, Germany
}

The blood-brain barrier (BBB) regulates passage of nutrients and signaling molecules from the circulation into the brain. Whether lipoproteins cross the BBB in vivo has been controversial, and no clear requirement for circulating lipoproteins in brain development has been shown. We address these issues in Drosophila, which has an functionally conserved BBB, and lipoproteins that resemble those of vertebrates. We show that the Drosophila lipoprotein lipophorin exists in two isoforms. Both isoforms cross the BBB, but accumulate on distinct subsets of cells within the brain. In addition to acting as a lipid carrier, lipophorin carries both sterol-linked and GPI-linked proteins into the circulation and transports them across the BBB. Finally, lipophorin promotes neuroblast proliferation by a mechanism that does not depend on delivery of dietary lipids. Transport of lipophorin and its cargo across the BBB represents a novel mechanism by which peripherally synthesized proteins might enter the brain and influence its development. Furthermore, lipid-linkage may be an efficient method to transport therapeutic molecules across the BBB.

\section{Introduction}

The BBB prevents unregulated passage of circulating molecules into the brain, maintaining a controlled and stable environment important for neuronal function (Banerjee and Bhat, 2007). To enter the brain, nutrients and signaling molecules must engage specific transport systems on BBB cells (Broadwell, 1989; Hervé et al., 2008). Lipoproteins mediate interorgan transport of nutritional and signaling lipids, and also bear a variety of lipid-linked signaling molecules (Panáková et al., 2005). Vertebrate BBB cells express lipoprotein receptors, and transcytose both LDL and HDL in tissue culture (Dehouck et al., 1997; Balazs et al., 2004). Furthermore, linking peptides derived from ApoE and ApoB to other molecules can promote their transport across the $\mathrm{BBB}$ (Spencer and Verma, 2007; Zensi et al., 2009). However, circulating lipoproteins have never been shown to enter to the brain in vivo. If lipoproteins do enter the brain, it is unclear what their function there would be since the brain does not use fatty acids for energy production (Yang et al., 1987). Furthermore, almost all cholesterol in the vertebrate brain is derived from endogenous synthesis rather than lipoprotein-mediated delivery (Dietschy and Turley, 2004; Dietschy, 2009).

The sterol auxotroph Drosophila melanogaster provides a powerful model system to study lipoproteins and the BBB. Their lipoproteins and lipoprotein receptors resemble those of vertebrates (van der Horst et al., 2002; Van der Horst et al., 2009;

Received Dec. 1, 2009; revised April 28, 2010; accepted June 3, 2010.

This work was supported by a European Molecular Biology Organization postdoctoral fellowship to M.B., by a grant from the Deutsche Forschungsgemeinschaft to S.E., and by the Max Planck Gesellschaft. We are grateful to Volker Hartenstein, Elisabeth Knust, Kai Simons, Wilhelm Palm, Christina Eugster, Andreas Sagner, and Daiki Umetsu for helpful discussions and critical comments on the manuscript. We thank Sven Ssykor for help with transgenesis and stock maintenance.

Correspondence should be addressed to Marko Brankatschk, Max Planck Institute of Molecular Cell Biology and Genetics (MPI-CBG), Pfotenhauer Strasse 108, 01307 Dresden, Germany. E-mail: brankats@mpi-cbg.de.

DOI:10.1523/JNEUROSCI.5943-09.2010

Copyright $\odot 2010$ the authors $\quad 0270-6474 / 10 / 3010441-07 \$ 15.00 / 0$
Khaliullina et al., 2010) and the functionally conserved BBB is formed by septate junctions composed of molecules almost identical to those in vertebrate tight junctions (Awasaki et al., 2008; Stork et al., 2008; Mayer et al., 2009). Here, we investigate whether the Drosophila lipoprotein lipophorin (Lpp) crosses the larval BBB, and whether it influences larval brain development.

\section{Materials and Methods}

\section{Cloning}

Lipophorin:GAL4. gal4 cDNA was inserted between the $5.5 \mathrm{kbp}$ promoter and $3 \mathrm{kbp} \mathrm{3'}$ ' UTR sequence of lipophorin and cloned into a vector containing a multiple cloning site and mini-white gene flanked by FRT sites.

Tagged mCherry reporters. An $840 \mathrm{bp}$ fragment containing the predicted signal sequence of lipophorin was fused to a HA-epitope triplet and cloned $5^{\prime}$ prime to mcherry to generate secreted ${ }^{\mathrm{HA}}{ }^{\mathrm{mCh}}$ Chry ${ }^{\mathrm{Sec}}$. For ${ }^{\mathrm{HA}}$ mCherry ${ }^{\mathrm{GPI}}$, we fused ${ }^{\mathrm{HA}}$ cherry ${ }^{\mathrm{Sec}}$ to LFA3-derived sequences specifying GPI addition. For ${ }^{\mathrm{HA}} \mathrm{mCherry}{ }^{\mathrm{Sterol}}$, we fused ${ }^{\mathrm{HA}}$ cherry $^{\mathrm{Sec}}$ to sequences encoding amino acids $243-472$ of $\mathrm{Hh}$. For ${ }^{\mathrm{HA}} \mathrm{mCherry}$, we fused ${ }_{\text {HA }}$ cherry $^{\text {Sec }}$ to the CD8 transmembrane domain sequence and inserted 2 $m y c$ tags $5^{\prime}$ to and a $v 5$ tag $3^{\prime}$ to the transmembrane domain. ${ }^{\mathrm{HA}}$ mCherry reporter constructs were cloned into pUAST.

Tagged tevP. To generate cytoplasmic TevP, the tevP cDNA was cloned into pUAST. To generate a TevP localized to the secretory pathway, we inserted the Lpp translational start site and signal sequence, and 3 HA-epitope tags $5^{\prime}$ of tevP.

\section{Fly stocks}

Flies were raised on conventional cornmeal agar under a $12 \mathrm{~h}$ light $/ 12 \mathrm{~h}$ dark cycle at $25^{\circ} \mathrm{C}$. UAS-lipophorinRNAi fly lines are from the Vienna Drosophila RNAi Center and UAS-Dicer2 flies are from Bloomington Stock Center. The ${ }^{H A} m$ Cherry ${ }^{\text {Sterol }}$ enhancer trap harbors a UAS ${ }^{H A} m-$ Cherry ${ }^{\text {Sterol }}$ construct expressed independently of GAL4.

\section{Collections}

Embryos collected at $4 \mathrm{~h}$ intervals were kept at $27^{\circ} \mathrm{C}$. Wild-type and $L p p G A L /+; U A S ; d s L p p /+$ larvae were transferred to applejuice agar plates containing yeast. Wild-type larvae were also transferred to lipiddepleted medium (Panáková et al., 2005). For phospho-histoneH3 stain- 
A

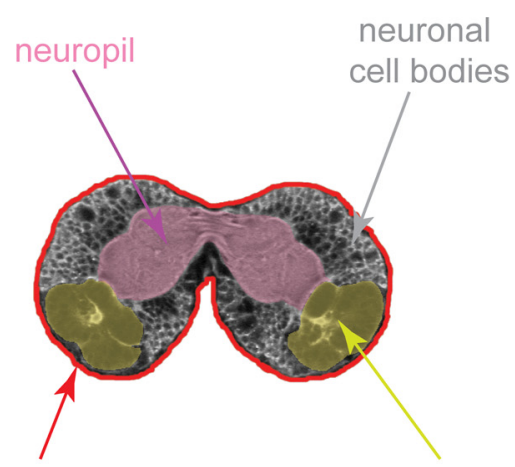

blood brain barrier

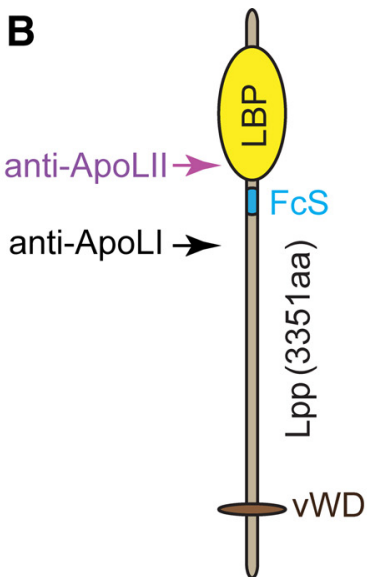

B

optic anlage
C \begin{tabular}{ll|l|l|l|l|l|l|l|l|l|}
\hline & 1.009 & 1.021 & 1.050 & 1.106 & 1.198 & 1.253 & 1.314 & 1.367 & $\mathrm{~g} / \mathrm{ml}$
\end{tabular}

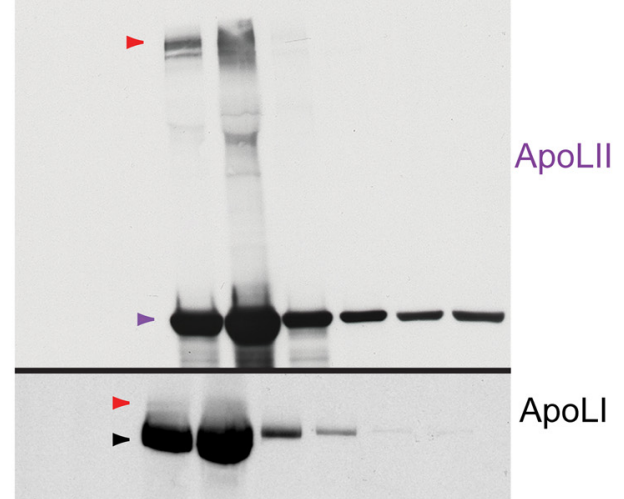

D

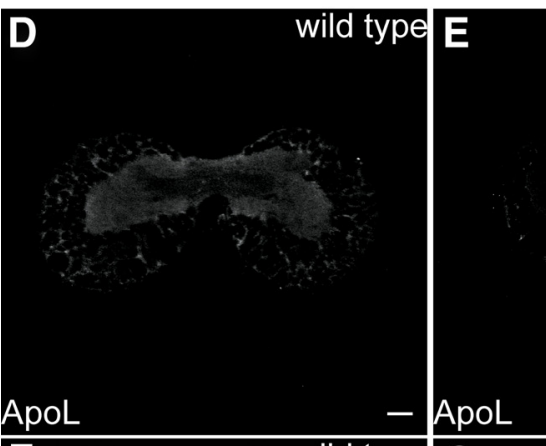

$\operatorname{Lpp} p^{R N A}$
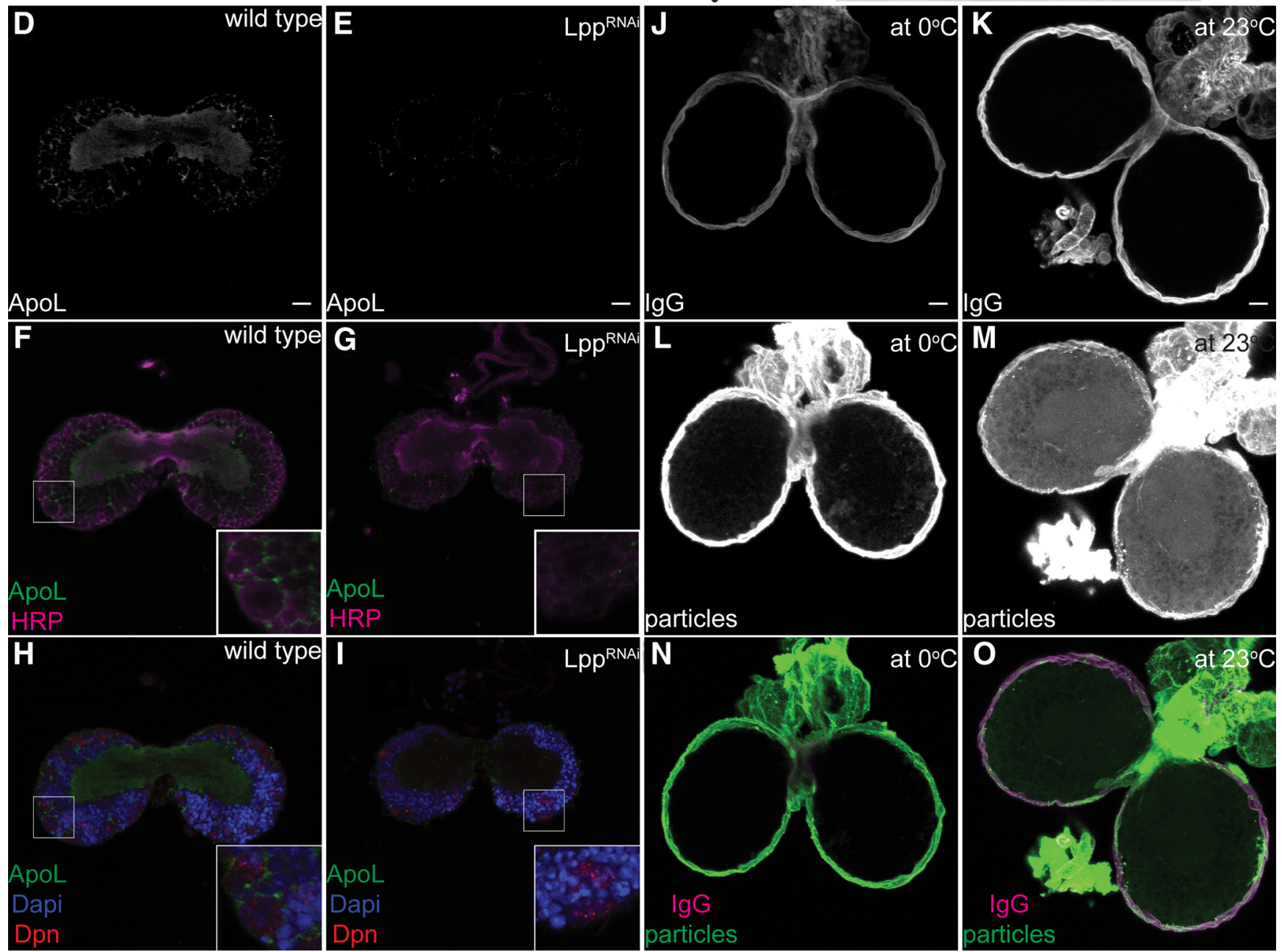

Figure 1. Lpp particles cross the larval BBB. $A$, Scheme depicts the larval CNS at the level of the commissure: neuropil (magenta), optic anlage (yellow), cell bodies (gray) and the BBB (red). $\boldsymbol{B}$, Motifs within apolipophorin. Lipid-binding pocket (LBP) (yellow oval), Furin cleavage site (FCS) (blue line), von Willebrand factor D domain (vWD) homology region (brown oval). Arrows indicate the position of peptides used to generate anti-ApoLII (magenta) and anti-ApoLI (black). C, Western blots of larval hemolymph iodixanol density gradients probed with anti-ApoLII and anti-ApoLI. Arrowheads indicate ApoLII (magenta), ApoLI (black) and Apo ${ }^{\mathrm{FL}}$ (red). Fraction densities in $\mathrm{g} / \mathrm{ml} . \boldsymbol{D}-\boldsymbol{I}$, Confocal section at the level of the commissures from wild-type $(\boldsymbol{D}, \boldsymbol{F}, \boldsymbol{H})$ and $\mathrm{Lpp}^{\mathrm{RNAi}}(\boldsymbol{E}, \boldsymbol{G}$, I) CNS stained with anti-Lpp $\left(\boldsymbol{D}, \boldsymbol{E} ; \boldsymbol{F}-\boldsymbol{I}\right.$; green), anti-HRP $\left(\boldsymbol{F}, \boldsymbol{G}\right.$; magenta), anti-Dpn $\left(\boldsymbol{H}, \boldsymbol{I} ;\right.$ red) and DAPI (4', $\mathbf{6}^{\prime}$-diamidino-2-phenylindole dihydrochloride) $(\boldsymbol{H}, \boldsymbol{I} ;$ blue). $\boldsymbol{J}-\mathbf{0}$, Wild-type larval brains incubated at $0^{\circ} \mathrm{C}(\boldsymbol{J}, \boldsymbol{L}, \boldsymbol{N})$ or $23^{\circ} \mathrm{C}(\boldsymbol{K}, \boldsymbol{M}, \mathbf{0})$ with labeled $L p p(\boldsymbol{L}, \boldsymbol{M} ; \boldsymbol{N}, \mathbf{0}$, in green) and labeled $\lg G(\boldsymbol{J}, \boldsymbol{K} ; \boldsymbol{N}, \mathbf{0}$; magenta). Ten micrometer projections at the level of the commissure.

ing, $L p p G A L /+; U A S ; d s L p p /+$ larvae and lipid-depleted wild-type larvae were collected after $5 \mathrm{~d}$. As normal second instar controls, we used wildtype larvae fed yeast-containing food for $41 \mathrm{~h}$.

\section{Hemolymph}

Larvae. Larvae were washed with ice-cold PBS, transferred into PBS + protease inhibitors and pierced with a tungsten needle. The hemolymph/
PBS was centrifuged $30 \mathrm{~min}, 550 \times g$ at $4^{\circ} \mathrm{C}$. The supernatant was recentrifuged $3 \mathrm{~h}, 112,000 \times g$ at $4^{\circ} \mathrm{C}$, and its protein concentration measured using the Bradford kit (Promega).

\section{Lpp labeling}

Fluorescent lipoproteins were prepared according to the method of Khaliullina et al. (2010). 
A

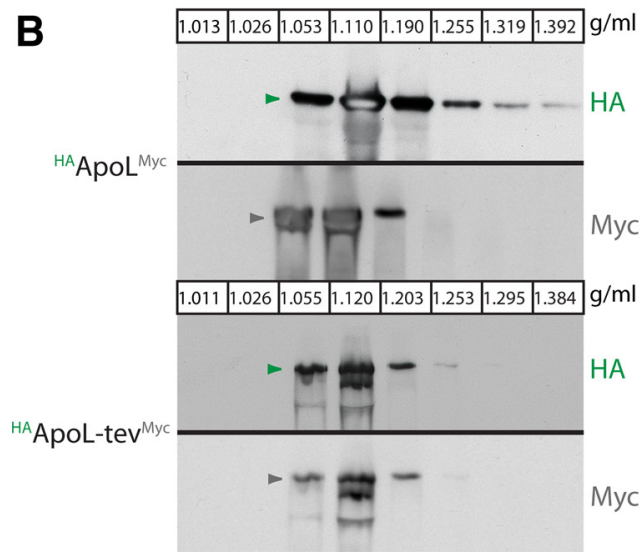

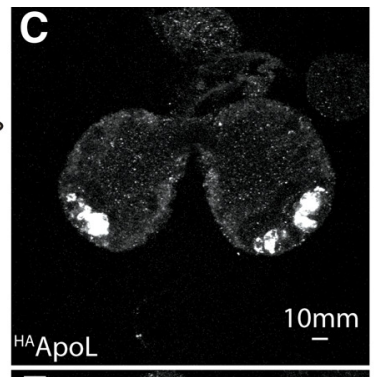
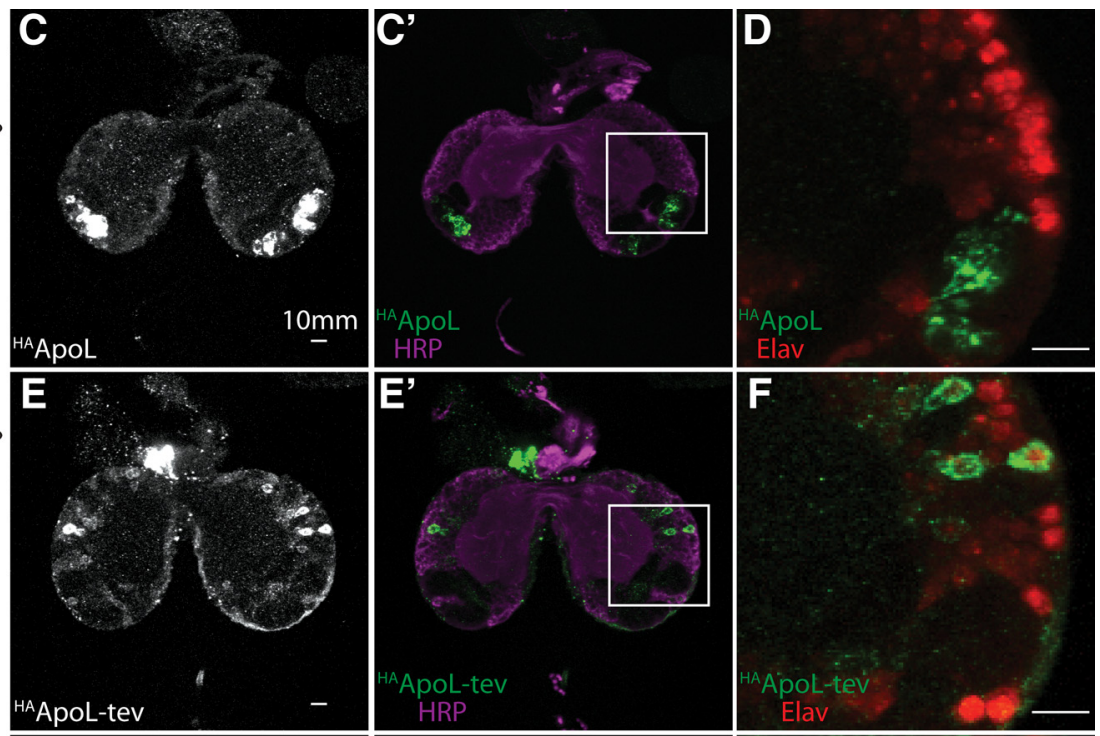

\section{G}
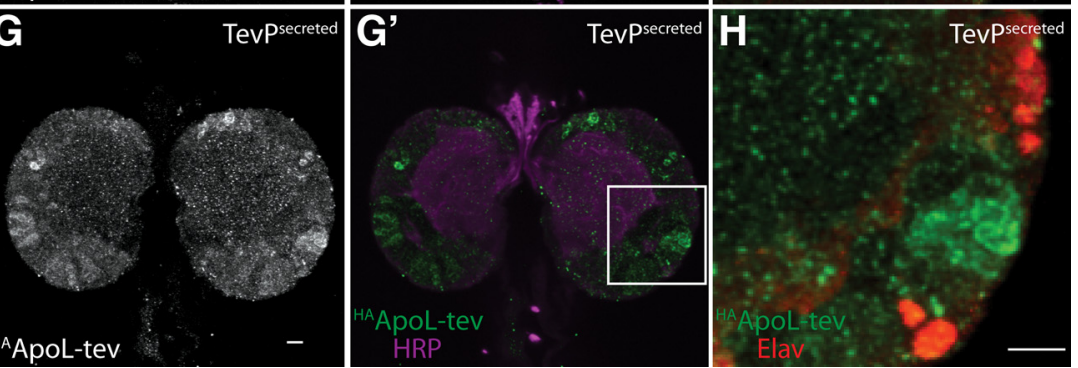

Figure 2. ${ }^{\mathrm{HA} A p o L-t e v}{ }^{\mathrm{Myc}}$ and ${ }^{\mathrm{HA}} \mathrm{ApoLIII} \mathrm{localize} \mathrm{differently} \mathrm{within} \mathrm{the} \mathrm{CNS.} \mathrm{A,} \mathrm{Replacement} \mathrm{of} \mathrm{the} \mathrm{apolipophorin} \mathrm{furin} \mathrm{cleavage} \mathrm{site} \mathrm{(blue)} \mathrm{with} \mathrm{TEV-protease} \mathrm{cleavage} \mathrm{sequences} \mathrm{(red).} \mathrm{HA} \mathrm{(green)}$ and Myc (gray) tags are shown. Other motifs as in Figure 1 B. B. Western blots of hemolymph iodixanol density gradients from larvae expressing either ${ }^{\mathrm{HA}} \mathrm{ApoL}{ }^{\mathrm{Myc}}$ or ${ }^{\mathrm{HA}} \mathrm{ApoL}-$ tev ${ }^{\mathrm{Myc}}$. Blots are probed with anti-HA (green arrowheads) and anti-Myc (gray arrowheads). Size heterogeneity of ${ }^{\mathrm{HA}} \mathrm{ApoL}$-tev ${ }^{\mathrm{Myc}}$ detected with both N-terminal HA and C-terminal Myc tags suggests alternative splicing may generate different ApoL $\mathrm{L}^{\mathrm{FL}}$ isoforms. Fraction densities in $\mathrm{g} / \mathrm{ml}$. $\mathbf{C}-\boldsymbol{H}$, Ten micrometer projections of second instar brains from larvae expressing either ${ }^{\mathrm{HA}} \mathrm{ApoL}{ }^{\mathrm{Myc}}\left(\boldsymbol{C}, \boldsymbol{C}^{\prime}, \boldsymbol{D}\right),{ }^{\mathrm{HA}} \mathrm{ApoL}-{ }_{\text {tev }}{ }^{\mathrm{Myc}}(\boldsymbol{E}$, $\left.\boldsymbol{E}^{\prime}, \boldsymbol{F}\right)$, or ${ }^{\mathrm{HA}} \mathrm{ApoL}-$ tev $^{\mathrm{Myc}}+\operatorname{TevP}^{\text {secreted }}\left(\boldsymbol{G}, \boldsymbol{G}^{\prime}, \boldsymbol{H}\right)$ in the fat body. Brains are stained with anti-HA $\left(\boldsymbol{C}, \boldsymbol{E}, \boldsymbol{G} ; \boldsymbol{C}^{\prime}-\boldsymbol{H} ;\right.$ green), anti-HRP $\left(\boldsymbol{C}^{\prime}, \boldsymbol{E}^{\prime}, \boldsymbol{G}^{\prime} ;\right.$ magenta) and anti-Elav $(\boldsymbol{D}, \boldsymbol{F}, \boldsymbol{H} ;$ red). Staining was observed only in the optic anlage for three of three brains expressing ${ }^{\mathrm{HA}} \mathrm{ApoL} \mathrm{L}^{\mathrm{Myc}}$. Both neuronal and optic anlage staining was observed for five of six brains from animals expressing ${ }^{\mathrm{HA}} \mathrm{ApoL}$-tev ${ }^{\mathrm{Myc}}+$ TevP ${ }^{\text {secreted }}$. Optic anlage staining was never observed in five of five brains from animals expressing either ${ }^{\mathrm{HA}} \mathrm{ApoL}-$ tev $^{\mathrm{Myc}}$ alone or $+\mathrm{TevP}$ cytoplasmic

\section{Immunohistochemistry}

Larval tissues dissected on ice in PBS were fixed with 4\%PFA at room temperature and stained in 10\% NGS, $0.1 \%$ Triton X-100 PBS solution. Antibodies were diluted as follows: anti-HA16B12 1:1500 (Santa Cruz Biotechnology), anti-Elav7E8A10 1:1500 [Developmental Studies Hybridoma Bank (DSHB), University of Iowa, Iowa City, IA], anti-HRPCy5 1:500 (Dianova), anti-Lpp (Eugster et al., 2007) 1:2000, anti-ApoLII 1:2000 and anti-ApoI 1:1500 (Panáková et al., 2005), anti-GFP 1:1000 (Invitrogen), anti-Ci ${ }^{155} 2 \mathrm{Al}$ (1:30) (Wang and Holmgren, 1999), anti-Dpn (1:30) (Boone and Doe, 2008), anti-Myc 1:1000 (Gramsch), anti-ProsperoMR1A 1:750 (DSHB), anti-Repo8D12 1:500 (DSHB) and anti-phosphohistone ${ }^{\mathrm{H} 3} 6 \mathrm{G} 3$ 1:1500 (Cell Signaling Technology).

Phalloidin-Cy5 1:200 (Invitrogen) and FilipinIII 1:500 (Sigma). For quantitation, tissues were treated in parallel and imaged under identical conditions on the same day using a Zeiss confocal microscope. Data were analyzed using Fiji.

\section{Quantification of CNS staining patterns}

To quantify accumulation of ${ }^{\mathrm{HA}} \mathrm{mCherry}{ }^{\mathrm{gpi}}$, ${ }^{\mathrm{HA}} \mathrm{mCherry}{ }^{\text {sterol }}$, and ${ }^{\mathrm{HA}}$ mCherry ${ }^{\mathrm{sec}}$ within the CNS, each section of a confocal stack comprising the entire CNS was examined by a blind observer, who enumerated Elav and HA-double-positive cells. To assess whether TEV-mediated cleavage of ${ }^{\mathrm{HA}} \mathrm{ApoL}-\mathrm{tev}{ }^{\mathrm{Myc}}$ increased accumulation in the optic anlage, a blind observer evaluated HA distribution in brains from animals expressing either ${ }^{\mathrm{HA}} \mathrm{ApoL}-\mathrm{tev}^{\mathrm{Myc}}$ alone or ${ }^{\mathrm{HA}} \mathrm{ApoL}-\mathrm{tev}^{\mathrm{Myc}}+\mathrm{TEV}$ protease in the fat body.

\section{Results}

To ask whether the major Drosophila lipoprotein, lipophorin (Lpp), accumulated in the larval brain, we stained brains with an antibody directed against Lpp particles. Lpp is detectable on the $\mathrm{BBB}$ and within the $\mathrm{CNS}$, suggesting these particles cross the $\mathrm{BBB}$ (Fig. 1A,D-I).

To directly observe passage of Lpp across the BBB, we used fluorescently labeled Lpp particles. Alexa543-labeled lipophorin and Alexa488-labeled IgG were added to explanted second instar larval brains and incubated at $0^{\circ} \mathrm{C}$. After $15 \mathrm{~min}$ at $0^{\circ} \mathrm{C}$, both Alexa543-labeled Lpp and Alexa488-labeled IgG accumulate on the BBB but not within the CNS (Fig. $1 J, L, N$ ). When the incubation is performed at $23^{\circ} \mathrm{C}$, Alexa488 $\mathrm{IgG}$ remains on the $\mathrm{BBB}$ and is still excluded from the CNS (Fig. $1 \mathrm{~K}, \mathrm{O}$ ). In contrast, Alexa543-lipophorin enters the brain and accumulates on neuronal cell bodies, axon tracts and cells of the optic anlage (Fig. $1 A, M, O)$. Thus, while IgG is efficiently excluded by the BBB, Lpp particles are actively transported across it.

The protein moiety of Lpp, apolipophorin, undergoes furindependent cleavage, generating apolipophorin I (ApoLI) and apolipophorin II (ApoLII) (Panáková et al., 2005), both of which circulate on low-density lipophorin particles in the hemolymph (Fig. $1 B, C$ ). Western blotting suggests that a fraction of circulating lipophorin particles also contains uncleaved apolipophorin, 
which we denote $\mathrm{ApoL}^{\mathrm{FL}}$ (Fig. 1C, red arrowheads). To investigate whether apolipophorin cleavage might influence its passage across the $\mathrm{BBB}$, we replaced the cleavage motif RXRR with a tobacco mosaic virus protease $(\mathrm{TevP})$ cleavage site triplicate (ApoL-tev). We tagged both wild-type apolipophorin $\left({ }^{\mathrm{HA}} \mathrm{ApoL} \mathrm{L}^{\mathrm{Myc}}\right.$ ) and ApoL-tev ( ${ }^{\mathrm{HA}} \mathrm{ApoL}-\mathrm{tev}^{\mathrm{Myc}}$ ) with HA at the $\mathrm{N}$ terminus and $\mathrm{Myc}$ at the $\mathrm{C}$ terminus (Fig. 2A) and expressed these constructs in the fat body (the normal site of lipophorin production) under the control of LppGAL4.

We first asked whether these tagged versions of apolipophorin were incorporated into lipophorin particles. Western blotting of larval hemolymph density gradients shows that ${ }^{\mathrm{HA}} \mathrm{ApoL}{ }^{\mathrm{Myc}}$ is normally processed to ${ }^{\mathrm{HA}} \mathrm{ApoLII}$ and ApoLI ${ }^{\mathrm{Myc}}$, which are incorporated into particles of the same density as wild-type Lpp (compare Fig. $2 B$, panels 1,2 with Fig. $1 C$ ). As expected, ${ }^{\mathrm{HA}} \mathrm{Apo}-\mathrm{tev}^{\mathrm{Myc}}$ is not detectably cleaved, and is incorporated in its fulllength form into particles of the same density as wild-type ApoL ${ }^{\mathrm{FL}}$ (compare Fig. $2 B$, panels 3, 4 with Fig. $1 C$ ). Thus, both proteins produce Lpp particles of normal density - ${ }^{\mathrm{HA}} \mathrm{ApoL}{ }^{\mathrm{Myc}}$ produces Lpp particles containing mostly the cleaved isoforms and ${ }^{\mathrm{HA}} \mathrm{ApoL}-\mathrm{tev}^{\mathrm{Myc}}$ forms lipoproteins in which apolipophorin is uncleaved.

To ask whether these particles differ in their ability to cross the BBB, we expressed either ${ }^{\mathrm{HA}} \mathrm{ApoL}^{\mathrm{Myc}}$ or ${ }^{\mathrm{HA}} \mathrm{ApoL}-\mathrm{tev}^{\mathrm{Myc}}$ under the control of LppGAL4. LppGAL4 is active only in the fat body and has no ectopic activity in the brain (supplemental Fig. S1 $A, B$, available at www.jneurosci. org as supplemental material). Furthermore, neither ${ }^{\mathrm{HA}} \mathrm{ApoL}^{\mathrm{Myc}}$ nor ${ }^{\mathrm{HA}} \mathrm{ApoL}-\mathrm{tev}^{\mathrm{Myc}}$ is detected in the brain when LppGAL4 is not present (supplemental Fig. S1C, available at www.jneurosci.org as supplemental material). In contrast, when these constructs are expressed in the fat body, both ApoL isoforms are found inside the CNS. Strikingly, they localize to different regions. Uncleavable ${ }^{\mathrm{HA}}$ ApoL-tev ${ }^{\mathrm{Myc}}$ localizes to neurons, whereas lipoproteins containing ${ }^{\mathrm{HA}} \mathrm{ApoLII}$ are found almost exclusively in the optic anlage (Fig. 2C-F). To confirm that their different localization depends on apolipophorin cleavage, we induced cleavage of ${ }^{\mathrm{HA}} \mathrm{ApoL}-\mathrm{tev}^{\mathrm{Myc}}$ by co-expressing the TevP in the fat body. When TevP is expressed in a form that can enter the secretory pathway, localization of ${ }^{\mathrm{HA}} \mathrm{ApoL}-\mathrm{tev}^{\mathrm{Myc}}$ shifts toward the optic anlage (Fig. $2 G, G^{\prime}, \mathrm{H}$ ). These data show that different types of lipoproteins accumulate in specific regions of the brain after crossing the BBB.

The first instar neuropil is composed of arborized neuronal projections formed by primary neurons that arise during embryogenesis. The neuropil increases in volume during early larval stages as primary neurons further extend neurites and increase their arborization. At the same time, continuous division of neuroblasts in the cortex generates what will become new secondary neurons (Larsen et al., 2009). To assess requirements for lipophorins in the brain at this stage, we used RNA interference to reduce lipophorin production in the fat body (supplemental Fig. S2 A-C, available at www.jneurosci.org as supplemental material). Lpp ${ }^{\text {RNAi }}$ larvae arrest in the second instar and live many days without developing further. Anti-HRP staining reveals that the second instar CNS of these larvae remains smaller than that of stage-matched control animals, and that this results from a reduction in the volume occupied by neuronal cell bodies and the optic anlage (Fig. $3 A, C$ ). Surprisingly, the size of the neuropil is even greater than that of wild-type second instar larvae (Fig. 3F; supplemental Fig. S3C, $D$, available at www.jneurosci.org as supplemental material). Staining glial cells with anti-Repo indicates that glial cell number is normal in these brains (supplemental Fig. S3G,H, available at www.jneurosci.org as supplemental material). Together, these data suggest that $\mathrm{Lpp}^{\mathrm{RNAi}}$ may block proliferation of neuroblasts without affecting neurite growth from primary neurons.

To investigate this further, we stained the CNS of $L p p^{R N A i}$ and control animals with anti-phospho-histone $\mathrm{H} 3$, which detects cells in mitosis, and with anti-Deadpan (Dpn), which labels neuroblasts in the CNS (Boone and Doe, 2008). In the wild-type 
A

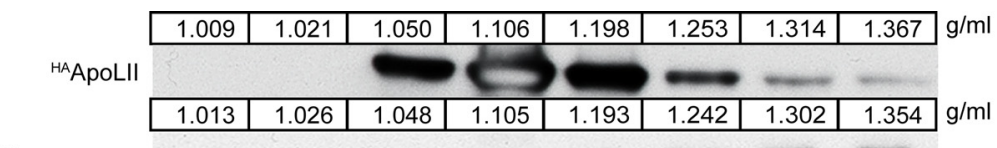

${ }^{H A}$ mCherrysec

${ }^{H A}$ mCherry ${ }^{\text {Ster }}$
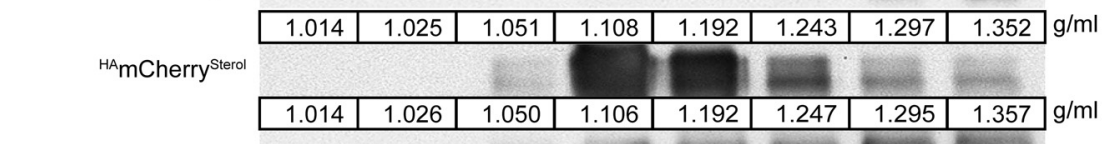

${ }^{\text {HA }}$ Cherry ${ }^{\text {Sterol }}$, Lpp $^{\text {RNA }}$

${ }^{H A}$ mCherry ${ }^{G P I}$

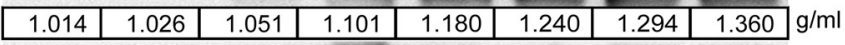

${ }^{H A}$ mCherry ${ }^{G P I}, L_{p p}^{\text {RNAi }}$
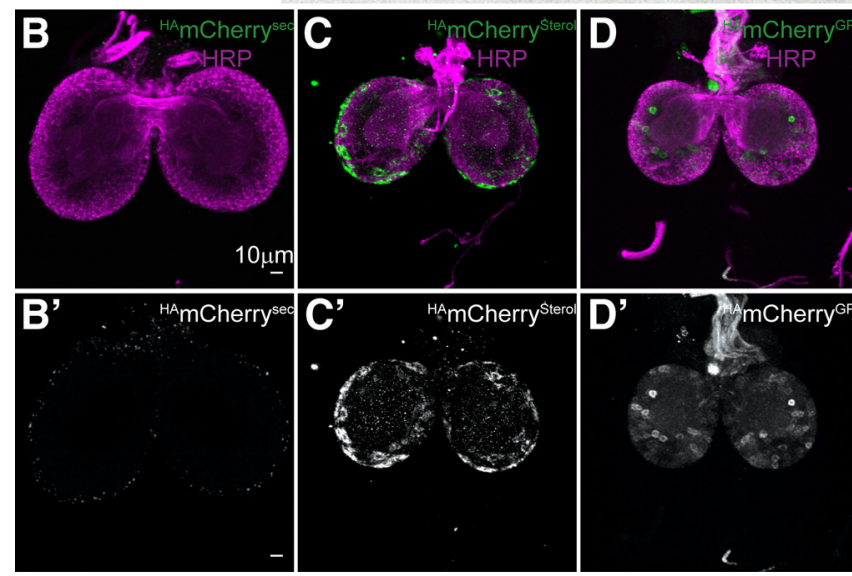

Figure 4. ${ }^{\mathrm{HA}} \mathrm{mCherry}{ }^{\mathrm{GPI}}$ crosses the BBB on Lpp. $\boldsymbol{A}$, lodixanol density gradients of hemolymph from third instar wild-type (rows $1,2,3,5)$ and $L p p^{\mathrm{RNAi}}$ larvae (rows 4,6) expressing ${ }^{\mathrm{HA}} \mathrm{mC}$ Cherry ${ }^{\mathrm{sec}}$, ${ }^{\mathrm{HA}} \mathrm{mCherry}{ }^{\text {sterol }}$ or ${ }^{\mathrm{HA}} \mathrm{mCherry}{ }^{\mathrm{GPI}}$ in the fat body. Western blots are probed with anti-ApoLII (first row) or anti-HA (rows 2-6). Fraction densities are shown in $\mathrm{g} / \mathrm{ml} . \boldsymbol{B}-\boldsymbol{D}^{\prime}$, Forty micrometer projection at the level of the commissures from wild-type $\left(\boldsymbol{B}, \boldsymbol{C}^{\prime}\right)$ and $\mathrm{Lpp}^{\mathrm{RNAi}}\left(\boldsymbol{D}, \boldsymbol{D}^{\prime}\right)$ second instar brains expressing ${ }^{\mathrm{HA}} \mathrm{mCherry}$ sec $\left(\boldsymbol{B}, \boldsymbol{B}^{\prime}\right),{ }^{\mathrm{HA}} \mathrm{mC}$ Cherry ${ }^{\text {sterol }}\left(\boldsymbol{C}, \boldsymbol{C}^{\prime}\right)$, or ${ }^{\mathrm{HA}} \mathrm{mCherry}{ }^{\mathrm{GPI}}\left(\boldsymbol{D}, \boldsymbol{E}^{\prime}\right)$ in the fat body stained with anti-HA $\left(\boldsymbol{B}^{\prime}, \boldsymbol{C}^{\prime}, \boldsymbol{D}^{\prime} ; \boldsymbol{B}-\boldsymbol{D} ;\right.$ green $)$ and anti-HRP $\left(\boldsymbol{B}-\boldsymbol{D}\right.$, magenta). ${ }^{\mathrm{HA}} \mathrm{mCherry}{ }^{\mathrm{sec}}$ is not found within the CNS ( ${ }^{\mathrm{HA}} \mathrm{mCherry}$-positive neurons in 10 brains examined). ${ }^{\mathrm{HA}} \mathrm{mCherry}{ }^{\mathrm{GPI}}$ accumulates in the CNS ( $>50{ }^{\mathrm{HA}} \mathrm{mCherry}$-positive neurons in all 5 brains examined). ${ }^{\mathrm{HA}} \mathrm{mCherry}{ }^{\text {sterol }}$ accumulates weakly in the CNS $\left(5,13\right.$, and $22{ }^{\mathrm{HA}} \mathrm{m}$ Cherry-positive neurons in 3 brains examined).

CNS, a significant fraction of Dpn-labeled neuroblasts also stain with anti-phospho-histone $\mathrm{H} 3$ (Fig. 3B). In contrast, proliferation in Lpp ${ }^{\text {RNAi }}$ brains is dramatically reduced (Fig. $3 E$ ). Although Dpn-positive neuroblasts are still detected in the CNS, they do not stain with anti-phospho-histone H3 (Fig. 3D; not a single Dpn-positive neuroblast stained with anti-phosphohistone $\mathrm{H} 3$ in $10 \mathrm{Lpp}^{\mathrm{RNAi}}$ brains examined). The few dividing cells observed in these brains were not Dpn-positive (supplemental Fig. S3 $A, B$, available at www.jneurosci.org as supplemental material). Thus, Lpp ${ }^{\text {RNAi }}$ blocks proliferation of neuroblasts, preventing generation of secondary neurons within the CNS.

To confirm that neurite extension from primary neurons was unaffected in Lpp ${ }^{\mathrm{RNAi}}$ animals, we labeled a single neuron that projects to the mushroom body and quantified the average length of its primary projection (before the first branch point). The average length does not differ in wild-type and $\mathrm{Lpp}^{\mathrm{RNAi}}$ animals (supplemental Fig. S4 A, B, available at www.jneurosci.org as supplemental material). Furthermore, the subset of FasII-positive neuronal projections in Lpp ${ }^{\mathrm{RNAi}}$ larvae are larger than those of equally staged control animals (supplemental Fig. $S 4 C, D$, available at www.jneurosci.org as supplemental material), confirming that loss of Lpp does not reduce neurite growth from existing primary neurons. Because neurite growth demands the incorporation of large amounts of lipid into the plasma membrane
(Pfenninger, 2009), these observations suggest that membrane lipids are not limiting in Lpp ${ }^{\mathrm{RNAi}}$ animals.

Although Drosophila requires sterols for development, they cannot synthesize them and depend on nutritional sources (Clayton, 1964). Lpp ${ }^{\text {RNAi }}$ inhibits lipid mobilization from the gut (Panáková et al., 2005) and reduces sterol levels in the larval imaginal discs (Khaliullina et al., 2010). Removal of dietary sterol arrests larval growth and development in the first/second instar (M. Carvalho and S. Eaton, unpublished observations), similar to the arrest seen in $\mathrm{Lpp}^{\mathrm{RNAi}}$ larvae. Therefore, we wondered whether reduced proliferation in the brain might be an indirect consequence of the lack of sterols or other essential dietary lipids. To investigate this, we compared the effects of Lp$\mathrm{p}^{\mathrm{RNAi}}$ versus dietary lipid depletion. Staining larval brains with FilipinIII, to detect free sterols, reveals that dietary lipid depletion reduces brain sterol levels more than Lpp ${ }^{\text {RNAi }}$ (Fig. 3G-I) However, while Lpp ${ }^{\mathrm{RNAi}}$ reduces mitosis dramatically, dietary lipid depletion has less severe effects (Fig. 3E,F; supplemental Figure $\mathrm{S} 3 C, D$, available at www.jneurosci.org as supplemental material). Thus, reduced proliferation in the brain of $\mathrm{Lpp}^{\mathrm{RNAi}}$ animals is not a consequence of the lack of sterol or other dietary lipids.

Since Lpp did not appear to promote proliferation by delivering dietary lipid, we wondered about other possible cargo. We previously reported that lipid-linked proteins (including GPIlinked, cholesterol-modified and palmitoylated proteins) associate with Lpp, permitting their local mobilization (Panáková et al., 2005; Eugster et al., 2007). We wondered whether Lpp association might also allow release of lipid-linked proteins into systemic circulation. To investigate this, we constructed ${ }^{\mathrm{HA}} \mathrm{mCherry}$ fusion proteins that were either secreted ( ${ }^{\mathrm{HA}} \mathrm{mCherry}{ }^{\mathrm{sec}}$ ), or targeted to the membrane in different ways. Membrane association was conferred either by a transmembrane domain ( $\left.{ }^{\mathrm{HA}} \mathrm{mCherry}\right), \mathrm{GPI}$ linkage $\left({ }^{\mathrm{HA}} \mathrm{mCherry}{ }^{\mathrm{GPI}}\right.$ ) or cholesterol modification $\left({ }^{\mathrm{HA}} \mathrm{mCherry}{ }^{\mathrm{Sterol}}\right)$. We expressed these constructs in the fat body, and analyzed larval hemolymph to determine whether they were released into systemic circulation. Both ${ }^{\mathrm{HA}}$ mCherry ${ }^{\mathrm{sec}}$ and the two lipid-linked forms circulate systemically. While soluble proteins such as ${ }^{\mathrm{HA}} \mathrm{mCherry}{ }^{\mathrm{sec}}$ are present only in high-density fractions, the majority of ${ }^{\mathrm{HA}}$ mCherry ${ }^{\text {Sterol }}$ and ${ }^{\mathrm{HA}}$ mCherry ${ }^{\mathrm{GPI}}$ cofractionates with Lpp particles (Fig. 4A). As expected, ${ }^{\mathrm{HA}} \mathrm{mCherry}$ is not present in the hemolymph, although it is expressed at equivalent levels in the fat body (supplemental Fig. S1, available at www.jneurosci.org as supplemental material). Thus, GPI and sterol-linked proteins are released into systemic circulation on Lpp.

To ask whether Lpp was required for release of ${ }^{\mathrm{HA}} \mathrm{mCherry}{ }^{\text {Sterol }}$ and ${ }^{\mathrm{HA}} \mathrm{mCherry}{ }^{\mathrm{GPI}}$ into the hemolymph, we reduced Lpp levels in animals expressing either ${ }^{\mathrm{HA}}$ mCherry ${ }^{\mathrm{Sterol}}$ or ${ }^{\mathrm{HA}}$ mCherry ${ }^{\mathrm{GPI}}$ in the fat body. Lpp knock-down dramatically reduces the amount of 
lipid-linked ${ }^{\mathrm{HA}} \mathrm{mCherry}$ present in low-density fractions. (Fig. $4 A$, compare rows 3,5 with rows 4,6 ). This indicates that ${ }^{\mathrm{HA}} \mathrm{mCherry}{ }^{\text {Sterol }}$ and ${ }^{\mathrm{HA}} \mathrm{mCherry}{ }^{\mathrm{GPI}}$ are released on Lpp, when it is present, but that other release mechanisms operate in its absence.

To test whether Lpp particles might act as vehicles for the transport of lipid-linked proteins across the BBB, we asked whether expression of ${ }^{\mathrm{HA}}$ mCherry fusion proteins in the fat body resulted in their accumulation in the CNS. Although ${ }^{\mathrm{HA}} \mathrm{mCherry}{ }^{\mathrm{sec}}$ circulates systemically, it is not found within the CNS (Fig. $\left.4 B, B^{\prime}\right)$. In contrast, when ${ }^{\mathrm{HA}} \mathrm{mCherry}{ }^{\mathrm{GPI}}$ is expressed in the fat body, it is detected on subsets of neurons within the brain at varying levels (Fig. $4 D, D^{\prime}$ ). ${ }^{\mathrm{HA}}$ mCherry ${ }^{\mathrm{GPI}}$ accumulates only on neurons, and not in the optic anlage-similar to the localization of Lpp particles containing uncleaved ${ }^{\mathrm{HA}} \mathrm{Apo-tev}{ }^{\mathrm{Myc}}$. The presence of ${ }^{\mathrm{HA}} \mathrm{mCherry}{ }^{\mathrm{GPI}}$ in the brain does not result from GAL4independent expression (supplemental Fig. S1 D,E, available at www.jneurosci.org as supplemental material). Interestingly, although ${ }^{\mathrm{HA}}$ mCherry ${ }^{\text {Sterol }}$, like ${ }^{\mathrm{HA}}$ mCherry ${ }^{\mathrm{GPI}}$, circulates on Lpp particles it is distributed differently when the particles reach the CNS. ${ }^{\mathrm{HA}}$ mCherry ${ }^{\text {Sterol }}$ accumulates strongly on the BBB, while ${ }^{\mathrm{HA}}$ mCherry ${ }^{\mathrm{GPI}}$ does not. Furthermore, while ${ }^{\mathrm{HA}}$ mCherry ${ }^{\mathrm{GPI}}$ is detected on at least 50 neurons/brain, many fewer neurons are positive for ${ }^{\mathrm{HA}}$ mCherry ${ }^{\text {Sterol }}$ (Fig. $4 C, D^{\prime}$ ). Thus, ${ }^{\mathrm{HA}}$ mCherry ${ }^{\mathrm{GPI}}$ crosses the BBB more efficiently than ${ }^{\mathrm{HA}} \mathrm{mCherry}{ }^{\text {Sterol }}$, and the non-Lpp-associated ${ }^{\mathrm{HA}} \mathrm{mCherry}{ }^{\mathrm{sec}}$ does not cross the BBB at all.

${ }^{\mathrm{HA}} \mathrm{mCherry}{ }^{\mathrm{GPI}}$ circulates in two forms, one Lpp-associated, and one of a higher density. To determine whether Lpp association was required for ${ }^{\mathrm{HA}}$ mCherry ${ }^{\mathrm{GPI}}$ to cross the $\mathrm{BBB}$, we knocked down Lpp in animals expressing ${ }^{\mathrm{HA}} \mathrm{mCherry}{ }^{\mathrm{GPI}}$ in the fat body. Lpp knock-down reduces levels of ${ }^{\mathrm{HA}}$ mCherry ${ }^{\mathrm{GPI}}$ in the brain (compare Fig. $4 D, D^{\prime}, E, E^{\prime}$ ), suggesting that ${ }^{\mathrm{HA}}$ mCherry ${ }^{\mathrm{GPI}}$ can cross the BBB only when associated with Lpp. Together, our data indicate that lipid-linked proteins cross the BBB in a lipophorindependent manner.

\section{Discussion}

The possibility that lipoproteins cross the BBB has been suggested by the presence of lipoprotein receptors on BBB cells, and by the observation that peptides derived from ApoE and ApoB promote transport of linked proteins across the BBB (Balazs et al., 2004; Kreuter et al., 2007; Spencer and Verma, 2007; Candela et al., 2008; Zensi et al., 2009). Nevertheless, there has been no evidence to show that circulating lipoproteins cross the $\mathrm{BBB}$ in vivo, or what their function in the brain might be if they did.

We show that the Drosophila lipoprotein lipophorin crosses the $\mathrm{BBB}$, and is essential for neuroblast proliferation. Our data suggest this requirement is independent of its function in lipid delivery. What other roles might Lpp play in brain development? The finding that lipophorin transports GPI-linked proteins into the CNS suggests a new range of mechanisms by which systemic signals might influence the nervous system: peripheral growthpromoting signals might be transported into the brain on Lpp. This may be of particular relevance to lipid-modified growth factors such as the EGF-related Spitz (Pan and Kastin, 1999; Miura et al., 2006). Furthermore, once inside the brain, Lpp may act as it does in imaginal discs (Panáková et al., 2005) to help mobilize signaling molecules (e.g., axon guidance cues), which act over large distances (Brankatschk and Dickson, 2006; Charron and Tessier-Lavigne, 2007).

Our observations suggest remarkable specificity in the trafficking of lipophorin and lipophorin-associated proteins in the nervous system. Two isoforms of Lpp differing only in the processing of apolipophorin both cross the BBB but accumulate in different regions of the CNS. Furthermore, although both sterol and GPI-linked proteins associate with Lpp, they are transported across the BBB with different efficiencies. This may reflect association with functionally different lipoprotein subpopulations, or differences in trafficking at the BBB. The cell biological basis of this specificity will be fascinating to unravel. Furthermore, these observations raise the possibility that linking therapeutic molecules to lipids could facilitate transport across the $\mathrm{BBB}$ by promoting association with lipoproteins.

\section{References}

Awasaki T, Lai SL, Ito K, Lee T (2008) Organization and postembryonic development of glial cells in the adult central brain of Drosophila. J Neurosci 28:13742-13753.

Balazs Z, Panzenboeck U, Hammer A, Sovic A, Quehenberger O, Malle E, Sattler W (2004) Uptake and transport of high-density lipoprotein (HDL) and HDL-associated alpha-tocopherol by an in vitro blood-brain barrier model. J Neurochem 89:939-950.

Banerjee S, Bhat MA (2007) Neuron-glial interactions in blood-brain barrier formation. Annu Rev Neurosci 30:235-258.

Boone JQ, Doe CQ (2008) Identification of Drosophila type II neuroblast lineages containing transit amplifying ganglion mother cells. Dev Neurobiol 68:1185-1195.

Brankatschk M, Dickson BJ (2006) Netrins guide Drosophila commissural axons at short range. Nat Neurosci 9:188-194.

Broadwell RD (1989) Transcytosis of macromolecules through the bloodbrain barrier: a cell biological perspective and critical appraisal. Acta Neuropathol 79:117-128.

Candela P, Gosselet F, Miller F, Buee-Scherrer V, Torpier G, Cecchelli R, Fenart L (2008) Physiological pathway for low-density lipoproteins across the blood-brain barrier: transcytosis through brain capillary endothelial cells in vitro. Endothelium 15:254-264.

Charron F, Tessier-Lavigne M (2007) The Hedgehog, TGF-beta/BMP and Wnt families of morphogens in axon guidance. Adv Exp Med Biol 621:116-133.

Clayton RB (1964) The utilization of sterols by insects. J Lipid Res 15:3-19.

Dehouck B, Fenart L, Dehouck MP, Pierce A, Torpier G, Cecchelli R (1997) A new function for the LDL receptor: transcytosis of LDL across the blood-brain barrier. J Cell Biol 138:877-889.

Dietschy JM (2009) Central nervous system: cholesterol turnover, brain development and neurodegeneration. Biol Chem 390:287-293.

Dietschy JM, Turley SD (2004) Thematic review series: brain lipids. Cholesterol metabolism in the central nervous system during early development and in the mature animal. J Lipid Res 45:1375-1397.

Eugster C, Panáková D, Mahmoud A, Eaton S (2007) Lipoprotein-heparan sulfate interactions in the Hh pathway. Dev Cell 13:57-71.

Hervé F, Ghinea N, Scherrmann JM (2008) CNS delivery via adsorptive transcytosis. Aaps J 10:455-472.

Khaliullina H, Panáková D, Eugster E, Riedel F, Carvalho M, Eaton S (2010) Patched regulates Smoothened trafficking using lipoprotein-derived lipids. Development, in press.

Kreuter J, Hekmatara T, Dreis S, Vogel T, Gelperina S, Langer K (2007) Covalent attachment of apolipoprotein A-I and apolipoprotein B-100 to albumin nanoparticles enables drug transport into the brain. J Control Release 118:54-58.

Larsen C, Shy D, Spindler SR, Fung S, Pereanu W, Younossi-Hartenstein A, Hartenstein V (2009) Patterns of growth, axonal extension and axonal arborization of neuronal lineages in the developing Drosophila brain. Dev Biol 335:289-304.

Mayer F, Mayer N, Chinn L, Pinsonneault RL, Kroetz D, Bainton RJ (2009) Evolutionary conservation of vertebrate blood-brain barrier chemoprotective mechanisms in Drosophila. J Neurosci 29:3538-3550.

Miura GI, Buglino J, Alvarado D, Lemmon MA, Resh MD, Treisman JE (2006) Palmitoylation of the EGFR ligand Spitz by Rasp increases Spitz activity by restricting its diffusion. Dev Cell 10:167-176

Pan W, Kastin AJ (1999) Entry of EGF into brain is rapid and saturable. Peptides 20:1091-1098. 
Panáková D, Sprong H, Marois E, Thiele C, Eaton S (2005) Lipoprotein particles are required for Hedgehog and Wingless signalling. Nature 435:58-65.

Pfenninger KH (2009) Plasma membrane expansion: a neuron's Herculean task. Nat Rev Neurosci 10:251-261.

Spencer BJ, Verma IM (2007) Targeted delivery of proteins across the blood-brain barrier. Proc Natl Acad Sci U S A 104:7594-7599.

Stork T, Engelen D, Krudewig A, Silies M, Bainton RJ, Klämbt C (2008) Organization and function of the blood-brain barrier in Drosophila. J Neurosci 28:587-597.

van der Horst DJ, van Hoof D, van Marrewijk WJ, Rodenburg KW (2002) Alternative lipid mobilization: the insect shuttle system. Mol Cell Biochem 239:113-119.
Van der Horst DJ, Roosendaal SD, Rodenburg KW (2009) Circulatory lipid transport: lipoprotein assembly and function from an evolutionary perspective. Mol Cell Biochem 326:105-119.

Wang QT, Holmgren RA (1999) The subcellular localization and activity of Drosophila cubitus interruptus are regulated at multiple levels. Development 126:5097-5106.

Yang SY, He XY, Schulz H (1987) Fatty acid oxidation in rat brain is limited by the low activity of 3-ketoacyl-coenzyme A thiolase. J Biol Chem 262:13027-13032.

Zensi A, Begley D, Pontikis C, Legros C, Mihoreanu L, Wagner S, Büchel C, von Briesen H, Kreuter J (2009) Albumin nanoparticles targeted with Apo E enter the CNS by transcytosis and are delivered to neurones. J Control Release 137:78-86. 